\title{
Accurate Numerical Modeling of the TARA Reflector System
}

\author{
Alex Heldring, Juan M. Rius, Member, IEEE, Leo P. Ligthart, Fellow, IEEE, and Angel Cardama, Member, IEEE
}

\begin{abstract}
The radiation pattern of the large parabolic reflectors of the Transportable Atmospheric RAdar system (TARA), developed at Delft University of Technology, has been accurately simulated. The electric field integral equation (EFIE) formulation has been applied to a model of the reflectors including the feed housing and supporting struts, discretised using the method of moments. Because the problem is electrically large (the reflector has a diameter of $33 \lambda$ ) and nonsymmetrical, this lead to a badly conditioned linear system of approximately half a million unknowns. In order to solve this system, an iterative solver (generalized minimum residual method) was used, in combination with the multilevel fast multipole method. Because of the bad conditioning, the system could only be solved by using a huge preconditioner. A new block-incomplete $L U$ preconditioner (ILU) algorithm has been employed to allow for efficient out-of-computer core memory preconditioning.
\end{abstract}

Index Terms-01reflector antennas, integral equations, method of moments (MoM), multilevel fast multipole method (MLFMA), numerical modeling, preconditioning.

\section{INTRODUCTION}

$\mathbf{O}$ NE OF THE MAJOR projects that has been conducted recently within the International Research Centre for Telecommunications and Radar (IRCTR) of the Delft University of Technology (TUD), is Transportable Atmospheric RAdar system (TARA) [1]. The TARA project, financed by the Netherlands Technology Foundation constitutes the design and construction of a mobile measurement facility incorporating the latest developments in antenna and Frequency Modulated-Continuous Wave (FM-CW) technology. The purpose of the measuring facility is the study of atmospheric phenomena such as clouds, precipitation and clear air turbulence.

Manuscript received October 8, 2002; revised July 28, 2003. This work was supported in part by the International Research Centre for Telecommunications and Radar (IRCTR), Delft University of Technology (TUD), under project "Modeling of Scattering and Radiation Problems," in part by the "Departament d'Universitats Recerca i Societat de la Informació (DURSI)" of the "Generalitat de Catalunya" under "Distinció de la Generalitat de Catalunya per a la Promoció de la Recerca Universitaria," in part by the Spanish "Comisión Interministerial de Ciencia y Tecnología (CICYT)" under grant TIC 2001-2364-C01-01 and in part by the European Commission under Future and Emerging Technologies (FET) project IST-2001-33055.

A. Heldring was with the International Research Centre for Telecommunications and Radar (IRCTR), Delft University of Technology (TUD), 2600 AA Delft, The Netherlands. He is now with Department of Signal Theory and Communications (TSC), Universitat Politècnica de Catalunya (UPC), 08034 Barcelona, Spain.

J. M. Rius and A. Cardama are with Department of Signal Theory and Communications (TSC), Universitat Politècnica de Catalunya (UPC), 08034 Barcelona, Spain (e-mail: rius@

L. P. Ligthart is with the International Research Centre for Telecommunications and Radar (IRCTR), Delft University of Technology (TUD), 2600 AA Delft, The Netherlands.

Digital Object Identifier 10.1109/TAP.2004.831408

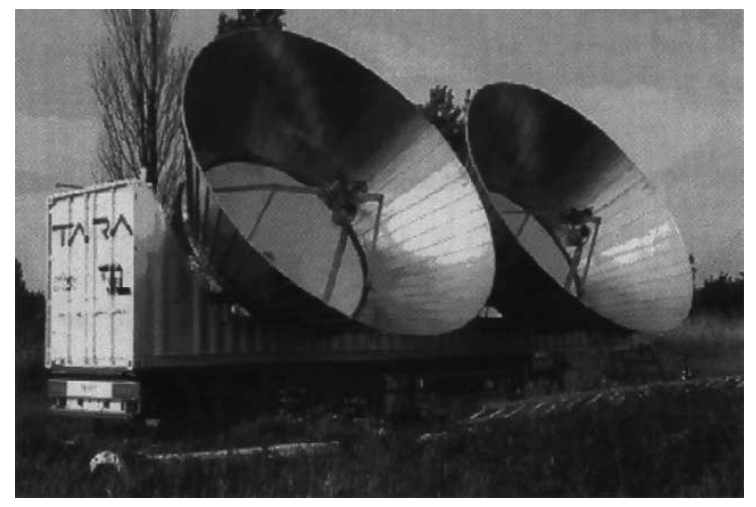

Fig. 1. TARA measurement facility in operation.

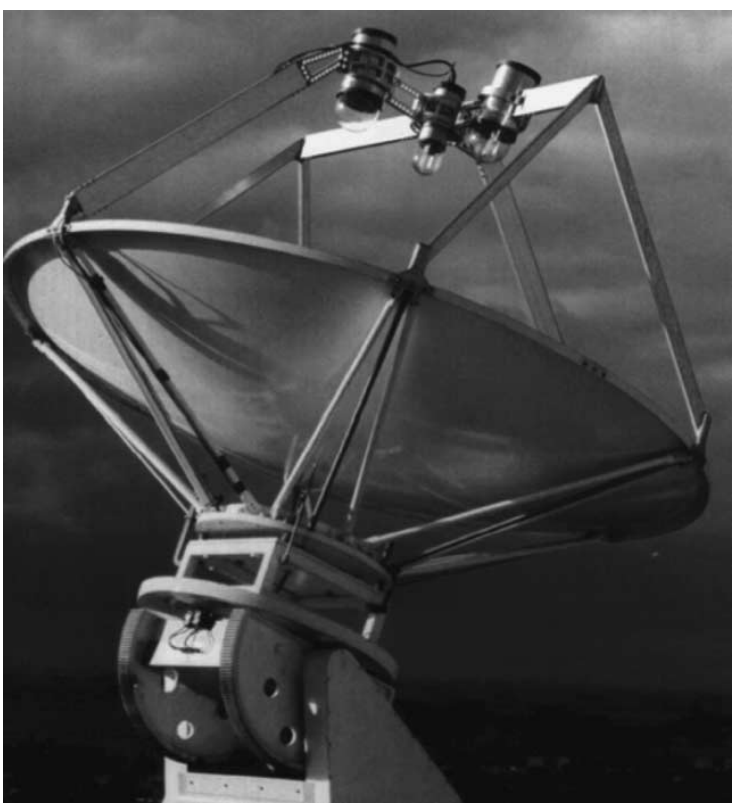

Fig. 2. TARA reflector in measurement setup.

The TARA project started in 1995 and the measurement facility has become operational in 2000. TARA consists of two large shielded parabolic reflector antennas, one for transmitting, one for receiving, mounted on a truck (see Fig. 1). The operating frequency is $3.3 \mathrm{GHz}$. The diameter of the reflectors is $3 \mathrm{~m}$, or approximately 33 wavelengths. The reflector antennas are fed by an advanced system of especially designed feed arrays, allowing for multiple beams in different directions and electronic beam-switching [2]. Fig. 2 shows a photograph of one of the two identical reflector antennas (without the shield), mounted in the IRCTR outdoor far field measurement range [3]. 
Because the system is mobile and meant to function at arbitrary locations, and because of the high sensitivity of the measurements, an essential requirement is a very high suppression of the interference from possible nearby obstacles. For this reason, one of the system specifications is a sidelobe level in the order of -70 to $-80 \mathrm{~dB}$ at $90^{\circ}$ from the main beam.

The usual approach to radiation pattern calculations of reflector antennas is to use high frequency techniques. Physical optics (PO) yields accurate results for the maximum gain and for the first few sidelobes. PO can be combined with physical theory of diffraction (PTD) to account for edge diffraction. But this approach fails to accurately incorporate the effect due to feed housings and struts. Alternatively, geometrical optics (GO) in combination with geometrical theory of diffraction (GTD) or uniform theory of diffraction (UTD) can be used. However, taking account of all the details of the TARA reflector becomes extremely complicated in practice with these methods.

The TARA problem requires a very high accuracy, in particular for the far sidelobes, which can only be provided by a full wave solution of the entire problem. Such a solution can be obtained with integral equation methods (IE) [4], in conjunction with method of moments (MoM) [5] discretization. The IE-MoM formulation leads to a full linear system of order $N$, where $N$ is the number of unknowns of the problem. The operation count for the solution grows with $N^{3}$ for direct solution (LU decomposition) or with $N^{2}$ (a matrix-vector product) per iteration for an iterative method.

The advent of very efficient methods for computing matrix-vector products in the iterative solution of the IE-MoM linear system has brought the solution of problems involving full matrices and a very large number of unknowns (several hundred thousands) within the reach of present computers. Examples of such efficient algorithms are the multilevel fast multipole algorithm (MLFMA) [6], [7], or the Multilevel matrix decomposition algorithm (MLMDA) [8], [9]. For a problem with a complex three dimensional (3-D) geometry, embedded in free space, the MLFMA is the most appropriate choice. It has been successfully applied to scattering problems with millions of unknowns [10], using the combined field integral equation, which leads to a well conditioned linear system of equations and thus allows for fast convergence of iterative solvers.

To our knowledge however, the MLFMA has not yet been applied to the problem of a large reflector antenna radiation pattern. The specific geometry of the TARA problem, a large and electrically thin metal surface (an open problem), calls for the use of the electric field integral equation (EFIE) instead of the CFIE, which cannot be applied to open problems. However, the EFIE impedance matrix has a much higher condition number that grows with the electrical size of the problem. Very large open problems like the TARA reflectors can only be solved with an iterative method if an efficient preconditioner is applied. Recently, a promising new technique has been proposed to precondition the EFIE [11], [12], but to the authors knowledge this new technique is not, in its present state of development, applicable to an open problem with nonsmooth boundaries, such as the TARA reflectors.
In this article, we present an MLFMA implementation with a new preconditioning scheme that allows to simulate the nonsymmetrical TARA reflectors with half a million unknowns on a desktop computer [13]. In Section II some aspects of our MLFMA implementation are presented. Concerning the theoretical background of the MLFMA, references to the relevant published literature are provided. In Section III we address the preconditioning problem and present our new preconditioning scheme. In Section IV, some numerical experiments are presented that illustrate the accuracy of MLMFA and the new preconditioning scheme. In the last section, our simulation results for the TARA radiation pattern are presented and compared to the available measured data.

\section{MLFMA}

We have implemented the MLFMA on top of a MoM code using a triangular patch model of the problem geometry and Rao, Wilton, and Glisson (RWG) basis functions [14]. In order to implement the MLFMA algorithm, the object must be subdivided into a set of nonoverlapping boxes at multiple levels in the form of an octal tree: the entire problem geometry is contained within a large cube, that is subdivided into eight cubes, which are subdivided into eight cubes again, and so on recursively, until a chosen minimum rib length is reached. The level of the largest cube is $l_{\max }$ and the smallest boxes are at level 1. The set of basis functions situated inside the same cube at a given level will be referred to as " group." At every level, the interactions between different groups are calculated using the fast multipole method (FMM) if the mutual distance between the cubes is larger than a given threshold parameter. At level 1 (smallest cubes), interactions between basis functions inside cubes that are too close together are computed in the conventional element by element fashion.

\section{A. FMM}

The FMM at the individual levels has been implemented according to the formulation given by Coifman et al., [6]. It involves a series expansion of the Green's function (the multipole expansion) and a transformation of the fields radiated by the groups to a plane wave expansion and back. The tradeoff between the accuracy and the efficiency of the FMM is governed by three parameters:

1) The number of terms $L$ in the multipole expansions;

2) The choice of the directions $\widehat{k}_{i}(i=1 \cdots K)$ in the plane wave expansion;

3) The minimum distance $R_{\min }$ between the cube centers.

The characteristic parameter for the groups is $D_{g}$, the diameter of the sphere enclosing the cubes, equal to $\sqrt{3}$ times the rib length. Coifman et al. [6] proposed the following empirical formula relating $D_{g}$ and $L$ :

$$
L \approx k D_{g}+P \log \left(k D_{g}+\pi\right)
$$

where $k$ is the wave number and $P$ is a constant, to be chosen as a function of the desired accuracy of the computation. This formula has been adopted and confirmed by various researchers. For very large groups $\left(k D_{g}>40\right)$, an alternative formula was 


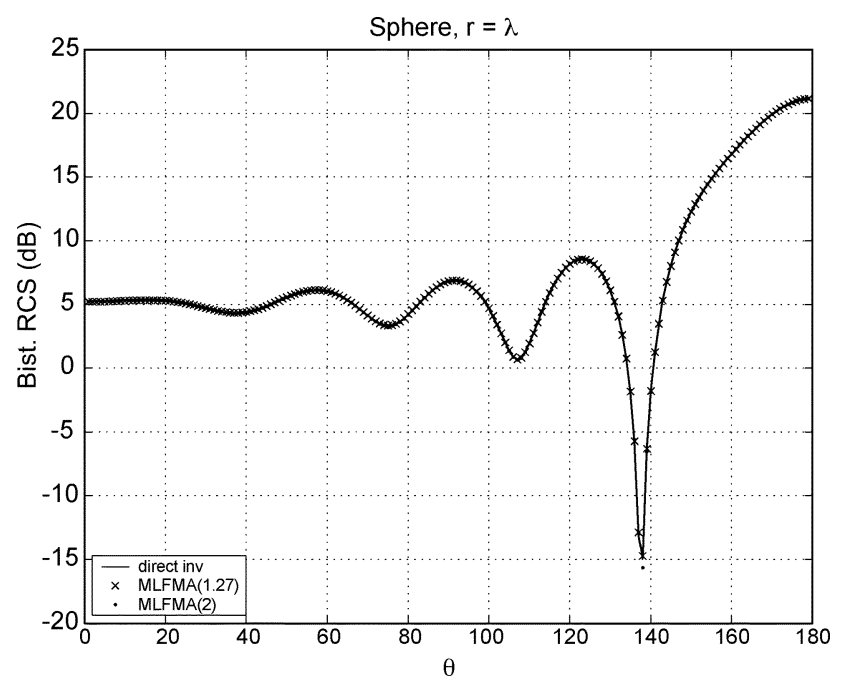

Fig. 3. Bistatic RCS of a PEC sphere, computed with MoM direct inversion, and with MLFMA using two different multipole-direction distributions: $\operatorname{MLFMA}(2)\left(K=2 L^{2}\right.$ azimuthal directions) and MLFMA(1.27) ( $K \sim 1.27 L^{2}$ azimuthal directions).

recently shown to be superior [15]. For the results presented in this paper however, the two formulas are equivalent.

For the distribution of the plane wave directions $\widehat{k}_{i}$ on the unit-sphere, Coifman et al., [6] proposed to take the zeros of $P_{L}(\cos \theta)$ (Legendre polynomial of order $L$ ) as abscissae in the $\theta$ direction, and $2 L$ evenly distributed points in $\phi$ direction. This choice guarantees to maintain the chosen accuracy. Gyure and Stalzer [16] show that it is exact for the set of spherical harmonics that form the base of the multipole expansion. The total number of directions then adds up to $K=2 L^{2}$. We have employed an adapted version of this distribution. We found experimentally that without loss of accuracy, one can reduce the number of points in $\phi$ direction depending on the latitude (value of $\theta$ ) and use $K_{\phi} \approx 2 L \sin (\theta)$ points instead of $2 L$ points. This reduces the number of directions asymptotically to

$$
K \sim 1.27 L^{2}
$$

yielding a nonnegligible gain in efficiency. Fig. 3 shows the bistatic RCS of a PEC sphere of radius $1 \lambda$, calculated using MoM with direct inversion, and using multilevel FMM (fivelevel MLFMA, see Section II-B), both with the $2 L^{2}$-distribution and with the $1.27 L^{2}$-distribution. The relative difference between the three curves is well below $-40 \mathrm{~dB}$ everywhere.

\section{B. Multilevel Implementation}

The number and distribution of the plane wave directions $K_{l}$ at level $l$ depends on the size of the cubes at that level. The key to an efficient implementation of the MLFMA is a method to obtain the plane wave representation of a group at level $l+1$ from that of its "children" (the eight cubes that make it up) at level $l$. This operation involves an interpolation and a shifting step [7]. The shifting step is straightforward. It shifts the origin of the plane wave representation from the child group centers to the parent group center. Darve [17] gives an overview of the proposed interpolation methods for the common $2 L^{2}$-distribution of multipole directions $\widehat{k}$. The interpolation can be made exact (without loss of information), but at a high price in com- putational efficiency. For the MLFMA to have a computational complexity of $N \log N$ the interpolation operator must be sparse and, therefore, approximate.

We have adopted a simple sequence of 1-D Lagrange interpolation schemes. In both the $\theta$ and the $\phi$ direction, the interpolation is obtained by fitting a polynomial of degree $w$ through the $w+1$ points that are nearest to the evaluation point. For the $1.27 K^{2}$-distribution this is a sequence of three steps. For interpolation between the levels $l$ and $l+1$ a sparse operator $\boldsymbol{\Phi}_{1}$ is applied that interpolates from the $\phi_{l}$ abscissa to an intermediary set of abscissa $\phi^{\prime}$ that are equal for all meridians. Then an operator $\Theta_{1}^{\mathbf{l}+\mathbf{1}}$ interpolates between the old and the new $\theta$-abscissa. Finally, an operator $\boldsymbol{\Phi}^{\mathbf{1 + 1}}$ interpolates from the $\phi^{\prime}$ to the new $\phi_{l+1}$. The entire interpolation operator

$$
\mathbf{W}_{1}^{1+1}=\Phi^{1+1} \Theta_{1}^{1+1} \Phi_{1}
$$

is a sparse $K_{l+1} \times K_{l}$ matrix with $\alpha K_{l+1}$ nonzeros, where $\alpha$ only depends on the degree of the interpolation.

Fig. 4 shows the nonzero patterns for $\mathbf{W}_{1}^{1+\mathbf{1}}$ for three different values of $w$. The construction of $\mathbf{W}_{1}^{1+\mathbf{1}}$ from its three factors causes little extra nonzero elements (fill-in). For example, for second degree interpolation (three points per row in the factors), the number of nonzero elements per row in $\mathbf{W}_{\mathbf{1}}^{\mathbf{l}+\mathbf{1}}$ converges to 11 for large group diameter.

In order to assess the accuracy of our implementation of the MLFMA, we have conducted a numerical experiment comparing the MLFMA to a direct LU decomposition of the full impedance matrix. As a testing problem we chose an infinitely thin rectangular perfectly conducting plate with dimensions $0.2 \lambda \times 6.4 \lambda$. This geometry allows for a direct solution because it can be modeled with relatively few unknowns $(N=318)$, but at the same time, since it spans several wavelengths, the MLFMA decomposition contains multiple levels $\left(l_{\max }=5\right)$. The plate was illuminated by a plane wave normal to the surface. We determined the relative difference

$$
\Delta_{\mathrm{j}}=\frac{\left|\mathbf{j}_{\text {direct }}-\mathbf{j}_{\text {MLFMA }}\right|}{\left|\mathbf{j}_{\text {direct }}\right|}
$$

where the $\mathbf{j}$ are vectors of RWG basis function coefficients representing the computed surface current. The relative error for RCS in the specular direction is computed similarly. The experiment was repeated for a sequence of values of the precision parameter $P$ and the interpolation degree $w$. The results are shown in Fig. 5. In our experience, the RWG model with a discretization size of around $0.1 \lambda$ has a relative accuracy for the far field in the range -20 to $-40 \mathrm{~dB}$, depending on the problem geometry. Clearly, it is useless to choose the MLFMA parameters such that the error due to MLFMA is several orders of magnitude lower than the error that is inherent to the RWG model, at a high computational cost. The results for the rectangular plate, shown in Fig. 5, indicate a choice of $P=2, w=4$ to keep the additional MLFMA error well below the $-40 \mathrm{~dB}$ level. However, reasonable results with an additional MLFMA error lower than - $30 \mathrm{~dB}$ can be obtained with the combination $P=1.5, w=2$. The above error estimates as a function of the precision parameter $P$ correspond well with the findings of other authors reporting an MLFMA error analysis [16]-[18]. 

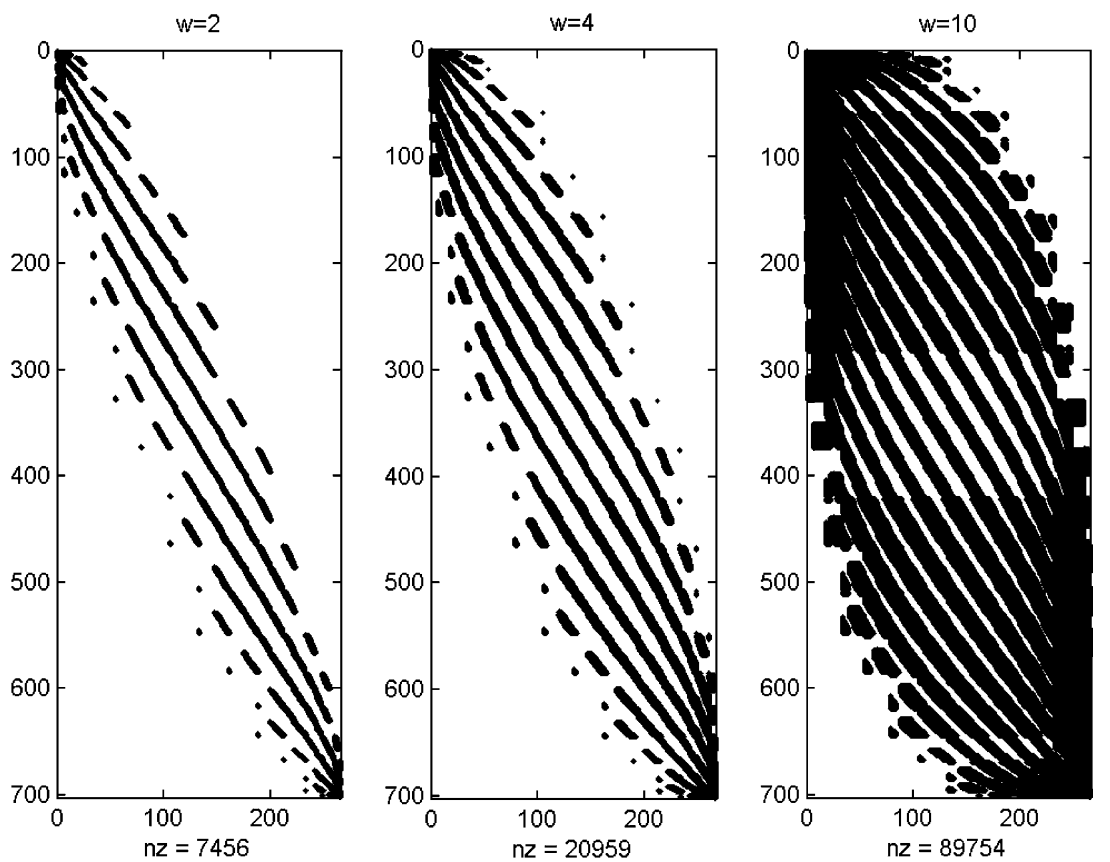

Fig. 4. Nonzero patterns of MLFMA interpolation matrices $(702 \times 266$ elements) for different degrees of Lagrange schemes $(2,4$, and 10$)$. The number of nonzeros per row is respectively $10.6,29.9$, and 127.9 .
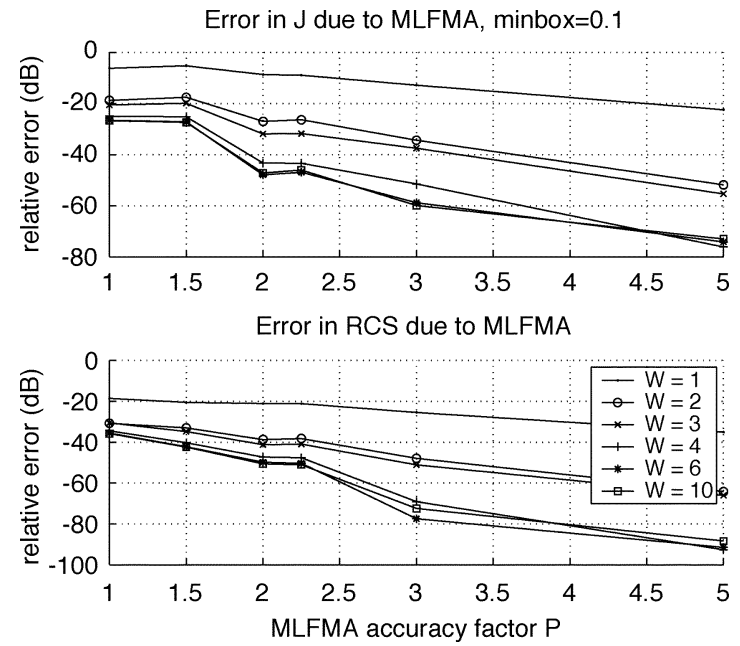

Fig. 5. Relative difference between direct solution and MLFMA solution for a $0.2 \lambda \times 6.4 \lambda$ perfectly conducting plate, for various values of the MLFMA precision factor $P$ and the interpolation degree $w$.

\section{PRECONDITIONING}

In order to solve iteratively the linear system resulting from MoM discretization, we have used the generalized minimum residual method (GMRES, [19]). The GMRES is a robust method for indefinite matrices like the EFIE impedance matrix. In every GMRES iteration step, the bulk of the effort consists of one matrix-vector multiplication of the impedance matrix with a working vector $\mathbf{x}$. The MLFMA accelerates the matrix-vector multiplication with an operation count proportional to $N \log N$, rather than the $N^{2}$ factor arising from conventional direct multiplication.

The iteration is stopped when

$$
\frac{|\mathrm{Zx}-\mathrm{e}|}{|\mathrm{e}|}<\epsilon_{r}
$$

where $\mathbf{e}$ is the excitation vector and $\epsilon_{r}$ is a given threshold, typically $1 \%$. However, the EFIE impedance matrix is generally badly conditioned, and the condition number grows with the electrical size of the problem. For large problems, the convergence can be very slow or even stagnate completely. The remedy is to use a left-preconditioner $\mathrm{M}^{-1}$, to transform the original linear system as follows

$$
\mathrm{Z} \mathbf{j}=\mathbf{e} \Rightarrow \mathrm{M}^{-1} \mathrm{Z} \mathbf{j}=\mathrm{M}^{-1} \mathbf{e}
$$

where $\mathbf{j}$ is the unknown surface current coefficient vector.

$\mathrm{M}^{-1}$ is chosen such that (a) it improves the total system condition number and (b) it is efficient to compute and to apply to the working vector. Condition (a) implies that $\mathrm{M}^{-1}$ should somehow approximate the inverse of Z. Condition (b) implies that both $\mathrm{M}$ and $\mathrm{M}^{-1}$ should be sparse or may be approximated by sparse matrices. It turns out that, in the case of the EFIE impedance matrix, we can obtain an efficient preconditioner by choosing for $\mathrm{M}$ a subset of those elements of $\mathrm{Z}$ that represent interactions between elements at a mutual distance smaller than a chosen radius $R_{\mathrm{M}}$. As with the MLFMA, an octal tree subdivision can be used to find these elements in an efficient way.

Subsequently, we build an incomplete LU preconditioner (ILU) [19], which is an LU factorization of $M$ with the elements on every row that are much smaller than the diagonal value dropped. The preconditioner is applied to the working vector $\mathbf{x}$ as

$$
\mathrm{M}^{-1} \mathbf{x} \approx \mathrm{U}^{-1} \mathrm{~L}^{-1} \mathbf{x}
$$

by forward and back substitution.

If the condition number were independent of the problem size, this preconditioner would scale linearly with $N$. For the EFIE however, large problems require larger $R_{\mathrm{M}}$ and lower threshold values in the ILU. Consequently, the size of M, L, and 
$\mathrm{U}$ grows fast. There is no theory available to estimate the relation between threshold values, sizes and convergence rate in a general case. For problems of the scale addressed in this paper $\left(N>1 \times 10^{5}\right)$, the typical size of $\mathrm{L}$ and $\mathrm{U}$ matrices is several Gigabytes in double precision. This poses a problem for the ILU factorization, since it needs simultaneous access to all elements in $\mathrm{M}, \mathrm{L}$, and $\mathrm{U}$. The conventional ILU algorithm is therefore limited to factorizations that fit in core memory.

We have developed a new block ILU algorithm that overcomes this problem, by partitioning the matrix $\mathrm{M}$ into several blocks, and performing the factorization on a block-by-block basis. This allows entire blocks to be swapped to and from hard disk storage, both in the factorization process and in the subsequent application phase.

\section{A. Block ILU Algorithm}

The proposed block ILU algorithm is based on the partitioned inverse formulas [20]. If a given matrix A is partitioned into four blocks

$$
\mathrm{A}=\left[\begin{array}{ll}
\mathrm{P} & \mathrm{Q} \\
\mathrm{R} & \mathrm{S}
\end{array}\right]
$$

where $\mathrm{P}$ and $\mathrm{S}$ are square submatrices, then the inverse can be expressed as

$$
\mathrm{A}^{-1}=\left[\begin{array}{ll}
\widetilde{\mathrm{P}} & \widetilde{\mathrm{Q}} \\
\widetilde{\mathrm{R}} & \widetilde{\mathrm{S}}
\end{array}\right]
$$

with

$$
\begin{aligned}
\widetilde{P} & =\left(P-\mathrm{QS}^{-1} \mathrm{R}\right)^{-1} \\
\widetilde{\mathrm{Q}} & =-\widetilde{\mathrm{P}}\left(\mathrm{QS}^{-1}\right) \\
\widetilde{\mathrm{R}} & =-\left(\mathrm{S}^{-1} \mathrm{R}\right) \widetilde{\mathrm{P}} \\
\widetilde{\mathrm{S}} & =\mathrm{S}^{-1}+\left(\mathrm{S}^{-1} \mathrm{R}\right) \widetilde{\mathrm{P}}\left(\mathrm{QS}^{-1}\right)
\end{aligned}
$$

as can be verified by substituting (10) in the product $\mathrm{AA}^{-1}$.

In order to use (10) recursively on the preconditioning matrix $\mathrm{M}$, it is subdivided into a chosen number of blocks of approximately equal sizes, based on the problem geometry. As a result M looks like

$\mathrm{M}=$\begin{tabular}{|c|c|c|c|c|}
\hline $\mathrm{P}_{n}$ & $\mathrm{Q}_{n}^{n-1}$ & $\mathrm{Q}_{n}^{n-2}$ & $\cdots$ & $\mathrm{Q}_{n}^{0}$ \\
\hline $\mathrm{R}_{n}^{n-1}$ & $\mathrm{P}_{n-1}$ & & & $\vdots$ \\
\hline $\mathrm{R}_{n}^{n-2}$ & & $\ddots$ & & \\
\hline$\vdots$ & & & $\mathrm{P}_{1}$ & $\mathrm{Q}_{1}^{0}$ \\
\hline $\mathrm{R}_{n}^{0}$ & $\cdots$ & & $\mathrm{R}_{1}^{0}$ & $\mathrm{~S}_{1}$ \\
\hline
\end{tabular}

where $n+1$ is the total number of blocks. Since M contains the largest elements in each row of the linear system matrix A and zeros elsewhere, with proper column and row ordering the diagonal blocks $P_{m}$ and $S_{1}$ are full or almost full, while many of the off-diagonal submatrices $\mathrm{Q}_{m}^{k}$ and $\mathrm{R}_{m}^{k}$ are very sparse. In the following, $\mathrm{Q}_{m}$ denotes the entire right-to-diagonal submatrix $\left[\mathrm{Q}_{m}^{m-1} \cdots \mathrm{Q}_{m}^{0}\right]$ and likewise $\mathrm{R}_{m}$ denotes the entire below-diagonal submatrix. Furthermore, $\mathrm{Q}_{m}^{(p)}$ denotes the block $\left[\mathrm{Q}_{m}^{p} \cdots \mathrm{Q}_{m}^{0}\right]$ and likewise for $\mathrm{R}_{m}^{(p)}$.
First, the ILU factorization of $\mathrm{S}_{1}$ is computed with a drop tolerance $\tau_{1}$. The operator $\left(U^{-1} L^{-1}\right)^{\prime}$ with $\mathrm{LU}=\mathrm{S}_{1}$ is represented by $\Sigma_{1}^{\prime}$, where the prime means that thresholding has been applied to drop small elements. $\Sigma_{1}^{\prime}$ is applied on the right to $\mathrm{Q}_{1}$ and on the left to $R_{1}$, yielding the factors

$$
\begin{aligned}
& \Theta_{1}=\mathrm{Q}_{1} \Sigma_{1}^{\prime} \\
& \Gamma_{1}=\Sigma_{1}^{\prime} \mathrm{R}_{1} .
\end{aligned}
$$

All elements below a chosen threshold $\tau_{2}$ with respect to the largest elements in $\Theta_{1}$ and $\Gamma_{1}$ are dropped (set to zero). The resulting matrices are denoted with $\Theta_{1}^{\prime}$ and $\Gamma_{1}^{\prime}$. Subsequently, the factor

$$
\Pi_{1}=\mathrm{P}_{1}-\mathrm{Q}_{1} \Gamma_{1}^{\prime} \approx \mathrm{P}_{1}-\mathrm{Q}_{1} \mathrm{~S}_{1}^{-1} \mathrm{R}_{1}
$$

is computed, and a dropping with threshold $\tau_{2}$ is applied to $\Pi_{1}$, yielding $\Pi_{1}^{\prime}$. Then, $\Pi_{1}^{\prime}$ is ILU factorized. The operator $\mathrm{U}^{-1} \mathrm{~L}^{-1}$ with $L U=\Pi_{1}^{\prime}$ is represented by $\widetilde{\mathrm{P}}_{1}$. The operators

$$
\widetilde{\mathrm{R}}_{1}=-\Gamma_{1}^{\prime} \widetilde{\mathrm{P}}_{1}
$$

and

$$
\widetilde{\mathrm{Q}}_{1}=-\widetilde{\mathrm{P}}_{1} \Theta_{1}^{\prime}
$$

are not explicitly stored, but whenever they are needed as operators on a matrix or vector, (15) and (16) are invoked. This concludes step one.

Now $\widetilde{\mathrm{P}}_{1}, \widetilde{\mathrm{Q}}_{1}$, and $\widetilde{\mathrm{R}}_{1}$ are available as operators, and so is $\Sigma_{1}^{\prime} \approx \mathrm{S}_{1}^{-1}$. Subsequently, the operators $\Gamma_{m}^{\prime}, \Theta_{m}^{\prime}$, and $\widetilde{\mathrm{P}}_{m}$ are computed and stored sequentially for $m=2 \cdots n$. $\Theta_{m}$ is defined as $\mathrm{Q}_{m} \Sigma_{m}$ and can be found with the recursive formula

$$
\mathrm{Q}_{p} \Sigma_{q}^{\prime}=\left[\left[\Xi_{q-1}^{p}\right]\left[-\Xi_{q-1}^{p} \Theta_{q-1}^{\prime}+\mathrm{Q}_{p}^{(q-2)} \Sigma_{q-1}\right]\right]
$$

in which

$$
\Xi_{q-1}^{p}=-\left(\mathrm{Q}_{p}^{q-1}-\mathrm{Q}_{p}^{(q-2)} \Gamma_{q-1}^{\prime}\right) \widetilde{\mathrm{P}}_{q-1} .
$$

Likewise, $\Gamma_{m}$ is defined as $\Sigma_{m} R_{m}$ and can be found with the recursive formula

$$
\Sigma_{q}^{\prime} \mathrm{R}_{p}=\left[\begin{array}{c}
\Lambda_{q-1}^{p} \\
-\Gamma_{q-1}^{\prime} \Lambda_{q-1}^{p}+\Sigma_{q-1} \mathrm{R}_{p}^{(q-2)}
\end{array}\right]
$$

in which

$$
\Lambda_{q-1}^{p}=\widetilde{\mathrm{P}}_{q-1}\left(\mathrm{R}_{p}^{q-1}-\Theta_{q-1}^{\prime} \mathrm{R}_{p}^{(q-2)}\right) .
$$

Every operation in (17) and (19) is followed by a dropping with threshold $\tau_{2}$, to obtain sparser $\Gamma_{m}^{\prime}$ and $\Theta_{m}^{\prime}$ from $\Gamma_{m}$ and $\Theta_{m}$. At every level, once $\Gamma_{m}^{\prime}$ is known, $\widetilde{\mathrm{P}}_{m}$ is computed as $\widetilde{\mathrm{P}}_{m}=$ $\mathrm{U}^{-1} \mathrm{~L}^{-1}$ with $\mathrm{LU}=\Pi_{m}^{\prime}$, where $\Pi_{m}^{\prime}$ is obtained from

$$
\Pi_{m}=\mathrm{P}_{m}-\mathrm{Q}_{m} \Gamma_{m}^{\prime}
$$

and a dropping with threshold $\tau_{2}$.

A close examination of (17) and (19) shows that the operators $\Sigma_{m}^{\prime}$ never have to be computed explicitly: for the computation of $\Gamma_{m}^{\prime}, \Theta_{m}^{\prime}$, and $\widetilde{\mathrm{P}}_{m}$ only $\Gamma_{k}^{\prime}, \Theta_{k}^{\prime}$, and $\widetilde{\mathrm{P}}_{k}$ with $k=$ $m-1, m-2 \cdots 1$ are needed. At each level $m$, all the previous factors are loaded and used recursively, down to level one. For 
symmetrical matrices like the EFIE impedance matrix, the factorization workload and storage is reduced by a factor of about one half because $\Gamma_{m}=\Theta_{m}^{\mathrm{T}}$. The choice of the threshold values $\tau_{1}$ and $\tau_{2}$ is a tradeoff between the effectiveness and the size of the preconditioner.

Once the preconditioner factors are computed, the preconditioner has to be applied at each iteration step to a working vector $\mathbf{x}$

$$
\mathbf{y} \approx \mathrm{M}^{-1} \mathbf{x} .
$$

The vector $\mathrm{x}$ is subdivided into $n+1$ blocks corresponding to the blocks of $\mathrm{M}$. Let $\mathrm{x}_{\mathrm{i}}$ denote the subset of elements corresponding to block $i$ and $\mathbf{x}_{(\mathrm{i})}$ the subset of elements corresponding to blocks 1 to $i$. The same notation applies to the vector y. For block one

$$
\mathbf{y}_{1}=\Sigma_{1}^{\prime} \mathbf{x}_{1} .
$$

The following blocks are computed recursively for $m=2 \cdots n+1$ with:

$$
\mathbf{y}_{(\mathrm{m})}=\left[\begin{array}{c}
\boldsymbol{\nu}_{\mathrm{m}} \\
-\Gamma_{m-1}^{\prime} \boldsymbol{\nu}_{\mathrm{m}}+\mathbf{y}_{(\mathrm{m}-1)}
\end{array}\right]
$$

where

$$
\boldsymbol{\nu}_{\mathrm{m}}=\widetilde{\mathrm{P}}_{\mathrm{m}-1}\left(\mathbf{x}_{\mathrm{m}}-\Theta_{\mathrm{m}-1}^{\prime} \mathbf{x}_{(\mathrm{m}-1)}\right)
$$

\section{NUMERICAL EXPERIMENTS}

In this section, a number of numerical experiments are presented in order to assess the accuracy of the computation. Firstly, in Section IV-A, the RCS of a $10 \lambda \times 10 \lambda$ infinitely thin perfectly conducting (PEC) square plate is computed, comparing three different methods: MLFMA with a conventional ILU preconditioner, MLFMA with a 16 block ILU preconditioner and MoM with direct inversion.

Secondly, in Section IV-B, the dependence of the MLFMA computation time and convergence on the problem size and on the angle of incidence is investigated. This comparison is made both with a conventional ILU preconditioner and with a 16 block ILU preconditioner. For this investigation, a $10 \lambda \times 10 \lambda$ square plate (the same as in Section IV-A), a $20 \lambda \times 20 \lambda$ square plate and a $40 \lambda \times 40 \lambda$ square plate are used.

The MLFMA group diameters $D_{g}$ are always equal to $\sqrt{3}$ times the rib length, as if the plates were 3-D objects.

Finally, in the next section, the radiation pattern of the TARA reflector is computed with the method presented in this paper and the result is compared with measurements. The computation times given for all the numerical experiments correspond to a personal computer with Athlon XP1900+ CPU.

\section{A. $10 \lambda \times 10 \lambda$ Square Plate}

A $10 \mathrm{~cm} \times 10 \mathrm{~cm}$ square plate was modeled into a mesh of right angled triangular patches, each triangle being one half of a $0.1 \mathrm{~cm} \times 0.1 \mathrm{~cm}$ patch. This led to a model consisting of $N=29800$ RWG basis functions. The incident field was a plane wave with a wavelength of $1 \mathrm{~cm}$, with the incidence direction perpendicular to the plate surface and the electric field parallel to two edges of the plate. The MLFMA was applied to
TABLE I

SiZE IN WAVELENGTHS, NUMBER OF UNKNOWNS (RWG BASIS FUNCTIONS) AND MLFMA LEVELS FOR THREE DIFFERENT INFINITELY THIN PEC SQUARE PLATES

\begin{tabular}{l|l|l|l}
\hline plate & A & B & C \\
\hline size $(\lambda)$ & $10 \times 10$ & $20 \times 20$ & $40 \times 40$ \\
\hline unknowns & 29800 & 119600 & 479200 \\
\hline levels & 7 & 8 & 9 \\
\hline
\end{tabular}

TABLE II

RWG/MLFMA/GMRES SURFACE CURRENT CALCULATION OF A $10 \lambda \times 10 \lambda$ Square Plate With Perpendicular $(\perp)$ and Parallel $(\|)$ Plane WaVe ILLUMINATION AND WITH TWO DIFFERENT PRECONDITIONERS

\begin{tabular}{c|c|c}
\hline \multicolumn{3}{c}{ plate A $(10 \lambda \times 10 \lambda)$} \\
\hline ILU & 1 block & 16 blocks \\
\hline size of M & $33 \mathrm{MB}$ & $33 \mathrm{MB}$ \\
\hline size of ILU & $137 \mathrm{MB}$ & $132 \mathrm{MB}$ \\
\hline ILU build time & $1 \mathrm{~min} 38 \mathrm{~s}$ & $2 \mathrm{~min} 45 \mathrm{~s}$ \\
\hline \multicolumn{3}{c}{ GMRES $\left(\epsilon_{r}<1 \%\right)$} \\
\hline step time & $8 \mathrm{~s}$ & $8 \mathrm{~s}$ \\
\hline \multicolumn{3}{c}{$\perp$ incidence } \\
\hline number of steps & 13 & 13 \\
\hline iteration time & 1 min $44 \mathrm{~s}$ & $1 \mathrm{~min} 44 \mathrm{~s}$ \\
\hline \multicolumn{3}{c}{$\|$ incidence } \\
\hline number of steps & 20 & 20 \\
\hline iteration time & 2 min $40 \mathrm{~s}$ & 2 min $40 \mathrm{~s}$ \\
\hline
\end{tabular}

this model, with the following parameters: precision parameter $P=1.5$ and degree of interpolation $w=2$. The side lengths of the smallest boxes in the MLFMA octal tree were set to $0.1 \mathrm{~cm}$. This resulted in a seven level algorithm.

A preconditioning matrix $\mathrm{M}$ was constructed, as described in Section III, with $R_{\mathrm{M}}=0.25 \lambda$. This lead to approximately 66 nonzero elements per row in M, occupying about $40 \mathrm{MB}$ of memory storage in double precision complex sparse storage format (approximately 20 bytes per element). From M, two different ILU decompositions were obtained, a conventional "one block" ILU and a 16 block ILU, using the algorithm of Section III-A. All the thresholds in the two ILU decompositions were set to 0.0025 .

The system was solved using GMRES both with the oneblock ILU preconditioner and with the 16 block ILU preconditioner. The stopping criterium for the GMRES was set to $1 \%$ for the relative residual. The matrix sizes are shown in Table I (column A) and the computation times for the two cases in Table II. These results show that the computation times and the effectivity of the two preconditioning methods are approximately equal.

The results of the two computations agreed well, with a $0.05 \%$ difference in the computed back scatter RCS. In order to validate the MLFMA model, the same problem was solved using the MoM with direct inversion. To this aim, the fourfold symmetry of the problem was exploited to reduce the number of unknowns to 7400. This was still too large for direct inversion, so the block ILU algorithm was invoked to build and invert the full impedance matrix on a block by block basis (16 blocks), setting all thresholds to zero. Building the matrix took $18 \mathrm{~min}$. and the decomposition took $41 \mathrm{~min}$. Fig. 6 gives the bistatic RCS of the square plate, for perpendicular incidence angle, as computed with MLFMA and MoM with direct block inversion. The relative difference at the maximum is $1.5 \%$. Finally, in order to illustrate the validity of the used distribution of 


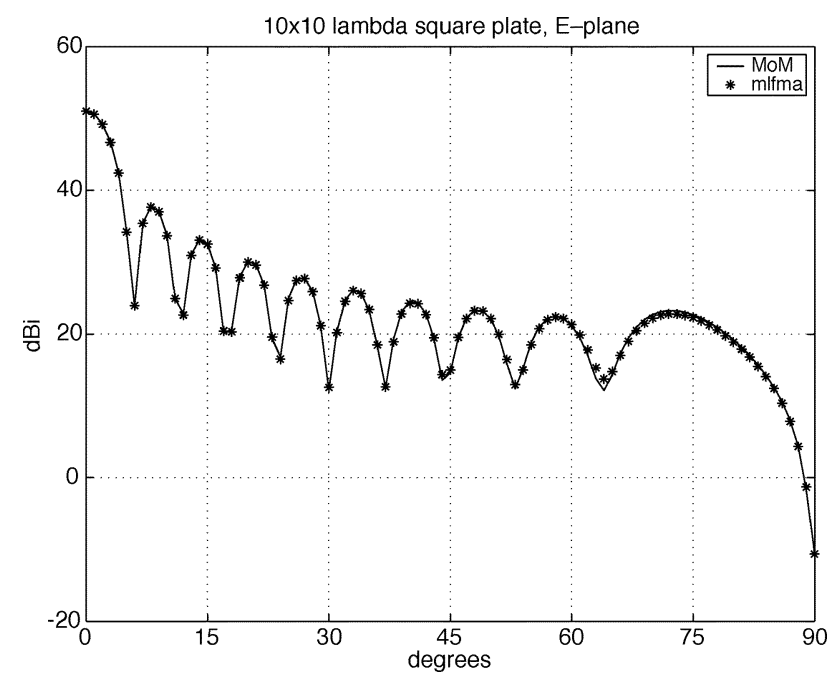

Fig. 6. Bistatic RCS in the E plane of a $10 \lambda \times 10 \lambda$ square plate with perpendicular incidence direction, computed with MLFMA and with MoM, direct inversion.

MLFMA plane wave directions (see Section II-A), the iterative solution with the one-block preconditioner was repeated twice, with the square plate positioned first in the $x-y$ plane and then in the $x-z$ plane, and the incident field rotated accordingly. This change affects the plane wave distribution for the MLFMA with respect to the plate surface. The results of the two computations agreed well, with a $0.1 \%$ difference in the computed back scatter RCS.

\section{B. Sequence of Square Plates}

In order to investigate the growth in computation time and required number of iteration steps to converge with growing problem size, the surface current has been computed for a sequence of square plates. The smallest plate was the same $10 \mathrm{~cm} \times 10 \mathrm{~cm}$ square plate from Section IV-A, followed by square plates with edge lengths $20 \mathrm{~cm}$ and $40 \mathrm{~cm}$. The illumination in all the computations was a $\lambda=1 \mathrm{~cm}$ plane wave. The computations were repeated for two different angles of incidence, perpendicular to the plate and parallel to the plate, with the electric field parallel to two edges of the plate in both cases. All computations were done with the GMRES/MLFMA method with the MLFMA parameters set to: precision parameter $P=1.5$ and degree of interpolation $w=2$, as in Section IV-A. The meshes were all constructed with the same triangle size, leading to a number of unknowns $N$ that grows with a factor of approximately four, as Table I shows. The three square plates were labeled plate A, B, and C, in the order of growing size.

In all three cases, the preconditioning matrix $\mathrm{M}$ was constructed as described in Section III with $R_{\mathrm{M}}=0.25 \lambda$. The computer requirements are summarized in Tables II-IV. The tables show that the size of M grows approximately linearly with $N$, as expected. All the preconditioners were built with threshold $\tau_{1}=0.0025$ and for all the 16 block preconditioners, a second threshold $\tau_{2}=0.0025$ was used. Only for the two smaller plates, a conventional (single block) ILU preconditioner was built. For the largest plate, this could not be done due to core memory constraints.
TABLE III

RWG/MLFMA/GMRES SURFACE CURRENT CALCULATION OF A $20 \lambda \times 20 \lambda$ Square Plate With Perpendicular $(\perp)$ and Parallel $(\|)$ Plane WaVe ILLUMINATION AND WITH TWO DIFFERENT PRECONDITIONERS

\begin{tabular}{c|c|c}
\hline \multicolumn{3}{c}{ plate B $(20 \lambda \times 20 \lambda)$} \\
\hline ILU & 1 block & 16 blocks \\
\hline size of M & $134 \mathrm{MB}$ & $134 \mathrm{MB}$ \\
\hline size of ILU & $564 \mathrm{MB}$ & $548 \mathrm{MB}$ \\
\hline ILU build time & $11 \mathrm{~min} 27 \mathrm{~s}$ & $16 \mathrm{~min} 40 \mathrm{~s}$ \\
\hline \multicolumn{3}{c}{ GMRES $\left(\epsilon_{r}<1 \%\right)$} \\
\hline step time & $38 \mathrm{~s}$ & $39 \mathrm{~s}$ \\
\hline \multicolumn{3}{|c}{$\perp$ incidence } \\
\hline number of steps & 16 & 17 \\
\hline iteration time & 10 min $8 \mathrm{~s}$ & $11 \mathrm{~min} 3 \mathrm{~s}$ \\
\hline & incidence \\
\hline number of steps & 41 & 41 \\
\hline iteration time & 25 min $58 \mathrm{~s}$ & $26 \mathrm{~min} 39 \mathrm{~s}$
\end{tabular}

TABLE IV

RWG/MLFMA/GMRES SURFACE CURRENT CALCULATION OF A $40 \lambda \times 40 \lambda$ Square Plate With Perpendicular $(\perp)$ and Parallel $(\|)$ Plane WaVe ILLUMINATION USING A 16 BLOCK ILU PRECONDITIONER

\begin{tabular}{c|c}
\hline \multicolumn{2}{c}{ plate C $(40 \lambda \times 40 \lambda)$} \\
\hline ILU & 16 blocks \\
\hline size of M & $541 \mathrm{MB}$ \\
\hline size of ILU $(\mathrm{MB})$ & $2.4 \mathrm{~GB}$ \\
\hline ILU build time & $4 \mathrm{~h} 1 \mathrm{~min}$ \\
\hline \multicolumn{2}{c}{ GMRES $\left(\epsilon_{r}<1 \%\right)$} \\
\hline step time & $250 \mathrm{~s}$ \\
\hline \multicolumn{2}{c}{$\perp$ incidence } \\
\hline number of steps & 21 \\
\hline iteration time & $1 \mathrm{~h} 28 \mathrm{~min}$ \\
\hline \multicolumn{2}{|c|}{ incidence } \\
\hline number of steps & 59 \\
\hline iteration time & $4 \mathrm{~h} 6 \mathrm{~min}$ \\
\hline
\end{tabular}

The total size of the ILU preconditioner (sum of all the blocks) grows linearly with $N$. However, the ILU decomposition time grows faster. There are two reasons for this: First, many elements are computed and then dropped, if their value is below the threshold level. This is a process which is impossible to predict because it depends on the actual composition of $\mathrm{M}$ and, therefore, on every individual problem. Second, in small problems a significant number of blocks are stored in memory, while in large problems almost all the blocks are stored in hard disk, thus saving a considerable amount of time if the entire preconditioner fits in core memory.

The time per GMRES step (iteration) is also given in the tables. The time per step includes the MLFMA matrix-vector product, the back and forward substitution of the preconditioner and a (small) overhead for the GMRES search-directions orthogonalization. Theoretically, this time grows with $N \log N$ (due to the MLFMA). Again, the times given in the tables are influenced by the number of blocks that are stored in memory or in hard disk.

The computations were done with two different incident field directions, perpendicular and parallel to the plate surface. The convergence of the GMRES iteration turns out to depend highly on the incident field. In the case of perpendicular incidence, the number of iterations increases very slowly with the size of the plate. In contrast, with parallel incidence the convergence is highly dependent on the size of the plate. 


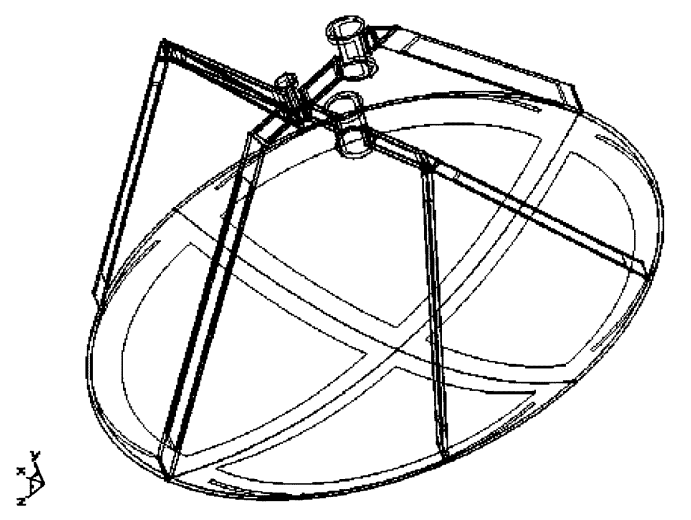

Fig. 7. Model of the TARA reflector with feeds and struts.

\section{TARA REFLECTOR RADIATION PATTERN}

We have modeled the TARA reflector system from Fig. 2 in detail using the meshing package GiD [22] developed at the International Center for Numerical Methods in Engineering (CIMNE), in Barcelona, Spain. The model is shown in Fig. 7. The parabolic dish has a diameter of $3 \mathrm{~m}$ and a focal distance of $1.535 \mathrm{~m}$, the wavelength being $9.1 \mathrm{~cm}$. The struts are metal beams with cross-sections of $12 \mathrm{~cm} \times 2.5 \mathrm{~cm}$ and $6 \mathrm{~cm} \times$ $2.5 \mathrm{~cm}$. The feed housings have been modeled as closed PEC cylinders. The central feed housing has a height of $24 \mathrm{~cm}$ and a diameter of $11.3 \mathrm{~cm}$. The off-axis feed housings have a height of $20 \mathrm{~cm}$ and a diameter of $26 \mathrm{~cm}$. They are tilted $6^{\circ}$ with respect to the focal axis. The feed housings, struts and the reflector surface at a distance smaller than $\lambda$ from the reflector edge were meshed with an average edgelength of $0.6 \mathrm{~cm}$. The rest of the reflector surface was meshed with an average edgelength of $1.2 \mathrm{~cm}$. This lead to $N=479200$ RWG basis functions (38\% of which represent the reflector surface, the rest is used for the feeds and supporting struts). The supporting structure underneath the reflector has been left out of consideration. The incident field used in the calculations was the measured radiation pattern of the central feed.

The induced surface current on the entire structure was computed using the MLFMA/GMRES method exposed in this paper, with the MLFMA parameters chosen to keep the error due to the MLFMA approximations in the far field below $1 \%$. The parameters, matrix sizes and computation times for this computation are summarized in Table V. The 40 block ILU preconditioner was built using thresholds $\tau_{1}=\tau_{2}=0.0025$. The largest block had a size of $150 \mathrm{MB}$. The GMRES iteration was stopped at a relative residual below $1 \%$ after 126 steps and the resulting surface current was used to calculate the radiation pattern. To make sure that the stopping criterium was sufficient, the iteration was interrupted at $\epsilon_{r}=2.01 \%$ after 106 steps, and the radiation pattern was also computed with the surface current solution at that stage. The two patterns were undistinguishable.

Fig. 8 shows the computed and measured radiation patterns for the E plane, with the feed polarization perpendicular to the long horizontal strut visible in Fig. 2. The measurements have been made at the IRCTR outdoor measurement facility. The results agree well qualitatively. The measured maximum gain was $38 \pm 0.5 \mathrm{dBi}$, the calculated maximum gain was $38.4 \mathrm{dBi}$. The sidelobe at approximately $-10^{\circ}$ is considerably higher in the
TABLE V

RWG/MLFMA/GMRES SURFACE CURRENT SOLUTION OF THE TARA REFLECTOR. THE COMPUTATION TIMES CORRESPOND TO A PERSONAL COMPUTER WITH ATHLON XP1900+ CPU

\begin{tabular}{|c|c|}
\hline \multicolumn{2}{|l|}{ RWG } \\
\hline number of unknowns & 479200 \\
\hline \multicolumn{2}{|l|}{ MLFMA } \\
\hline$P$ & 2 \\
\hline interpolation degree & 4 \\
\hline levels & 9 \\
\hline \multicolumn{2}{|c|}{40 block ILU } \\
\hline$R_{\mathrm{M}}$ & $0.35 \lambda$ \\
\hline size of $M$ & $8.4 \mathrm{~GB}$ \\
\hline size of ILU & $3.42 \mathrm{~GB}$ \\
\hline ILU build time & $4 \mathrm{~h} 45 \mathrm{~min}$ \\
\hline \multicolumn{2}{|c|}{ GMRES $\left(\epsilon_{r}<1 \%\right)$} \\
\hline step time & $8 \mathrm{~min} 47 \mathrm{sec}$ \\
\hline number of steps & 126 \\
\hline iteration time & 18 h $26 \mathrm{~min}$ \\
\hline tal computation time & $25 \mathrm{~h} 15 \mathrm{~min}$ \\
\hline
\end{tabular}

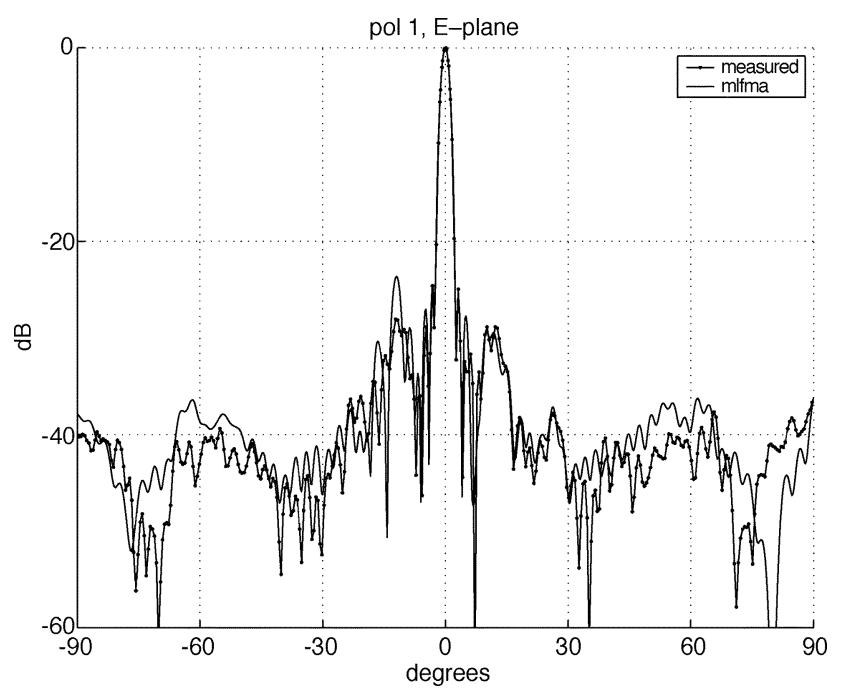

Fig. 8. Measured and computed radiation pattern (E plane) of the TARA reflector.

MLFMA simulation. This sidelobe is caused by a secondary reflection from the off-axis feed in the E plane. A possible explanation for the divergence between the computed and the measured result, is that the physical feed structure, consisting of an array of two dielectric rods, disperses the field whereas the flat metal surface assumed in the computational model causes a specular reflection in the observed direction.

\section{CONCLUSION}

We have presented a new block Incomplete LU preconditioner scheme that is as efficient as the well known conventional Incomplete LU preconditioner but, due to its block structure, allows the use of a preconditioner much larger than the available core memory. Indeed, the only limitation is hard-disk space. Using this new technique in combination with the MLFMA, we have been able to perform a full wave analysis of an electrically large reflector antenna, including all the relevant details. The results are presented and compared to measurements of the reflector antenna. 


\section{REFERENCES}

[1] S. Heijnen and L. P. Ligthart, "TARA: transportable atmospheric radar," in Proc. 28th Eur. Microwave Conf, 1998, p. 61.

[2] A. Moumen, "Analysis and Synthesis of Compact Feeds for Large Multiple-Beam Reflector Antennas," Ph.D. dissertation, Delft University of Technology, Delft, The Netherlands, 2001.

[3] P. Aubry, P. Hakkaart, J. H. Zijderveld, and L. P. Ligthart, "Implementation of an outdoor far-field measurement system at IRCTR," in Proc. 28th Eur. Microwave Conf., 1998, pp. 549-552.

[4] N. Morita, N. Kumagai, and J. R. Mautz, Integral Equation Methods for Electromagnetics. Norwood, MA: Artech House, 1990.

[5] R. F. Harrington, Field Computation by Moment Methods. New York: MacMillan, 1968.

[6] R. Coifman, V. Rohklin, and S. Wandzura, "The fast multipole method for the wave equation: a pedestrian description," IEEE Antennas Propagat. Mag., vol. 35, pp. 7-12, June 1993.

[7] J. Song, C. C. Lu, and W. C. Chew, "Multilevel fast multipole algorithm for electromagnetic scattering by large complex objects," IEEE Trans. Antennas Propagat., vol. 45, pp. 1488-1493, Oct. 1997.

[8] J. M. Rius, J. Parrón, E. Úbeda, and J. R. Mosig, "Multilevel matrix decomposition algorithm for analysis of electrically large electromagnetic problems in 3-D," Microwave Opt. Technol. Lett., vol. 22, no. 3, pp. 177-182, Aug. 1999.

[9] J. Parrón, J. M. Rius, and J. R. Mosig, "Application of the multilevel decomposition algorithm to the frequency analysis of large microstrip antenna arrays," IEEE Trans. Magn., vol. 38, pp. 721-724, Mar. 2002.

[10] S. Velamparambil and W. C. Chew, "Parallelization of MLFMA on distributed memory computers," in Proc. Int. Conf. Electromagnetics in Advanced Applications (ICEAA-01), Torino, Italy, Sept., 10-14 2001, pp. 141-144.

[11] R. J. Adams and G. S. Brown, "Stabilization procedure for electric field integral equations," Electron. Lett., vol. 35, pp. 2015-2016, Nov. 1999.

[12] H. Contopaganos et al., "Well-conditioned boundary integral equations for three-dimensional electromagnetic scattering," IEEE Trans. Antennas Propagat., vol. 50, pp. 1824-1830, Dec. 2002.

[13] A. Heldring, "Full wave analysis of electrically large reflecto antennas," Ph. D. dissertation, Delft Univ. Press, Delft, The Netherlands, Apr. 2002.

[14] R. M. Rao, D. R. Wilton, and A. W. Glisson, "Electromagnetic scattering by surfaces of arbitrary shapes," IEEE Trans. Antennas Propagat., vol. 30, pp. 409-418, May 1982.

[15] J. Song and W. C. Chew, "Error analysis for the truncation of multipole expansion of vector Green's functions," IEEE Microwave Wireless Components Lett., vol. 11, pp. 311-313, July 2001.

[16] M. F. Gyure and M. A. Stalzer, "A prescription for the multilevel Helmholtz FMM," IEEE Comput. Sci. Eng., pp. 39-47, July 1998.

[17] E. Darve, "The fast multipole method: numerical implementation," $J$. Comput. Phys., vol. 160, pp. 195-240, 2000.

[18] K. Sertel, M. Carr, and J. L. Volakis, "Error evaluation of fast integral methods for RCS computations," in Proc. Millennium Conf. Antennas and Propagation (AP2000), Davos, Switzerland, 2000.

[19] Y. Saad, Iterative Methods for Sparse Linear Systems. Boston, MA: PWS, 1996.

[20] W. H. Press et al., Numerical Recipes in C, 2nd ed. Cambridge, U.K.: Cambridge Univ. Press, 1994.

[21] S. H. Heijnen and L. P. Ligthart, "TARA: transportable atmospheric radar," in Proc. 28th Eur. Microwave Conf., vol. 1, 1998, pp. 61-66.

[22] R. Ribó et al., GiD Reference Manual. Barcelona, Spain: International Center For Numerical Methods in Engineering (CIMNE), 1998.

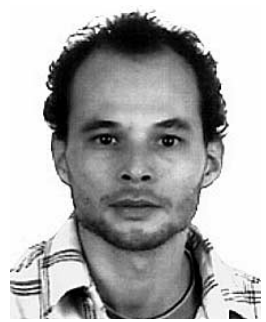

Alex Heldring was born in Amsterdam, The Netherlands, on December 12, 1966. He received the M.S. degree in applied physics and the Ph.D. degree in electrical engineering from the Delft University of Technology, Delft, The Netherlands, in 1993 and 2002 , respectively.

$\mathrm{He}$ is presently working as an Assistant Professor at the Telecommunications Department, Universitat Politecnica de Catalunya, Barcelona, Spain. His special research interests include integral equation methods for electromagnetic problems, preconditioning and wire antenna analysis.

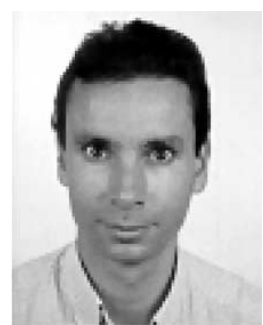

Juan M. Rius (S'89-M'91) received the "Ingeniero de Telecomunicación" degree and the "Doctor Ingeniero" degree from the Universitat Politècnica de Catalunya (UPC), Barcelona, Spain, in 1987 and 1991, respectively.

In 1985, he joined the Electromagnetic and Photonic Engineering group at UPC, where he currently holds a position of "Catedrático," equivalent to full Professor. From 1985 to 1988, he developed a new inverse scattering algorithm for microwave tomography in cylindrical geometry systems. Since 1989 , he has been engaged in the research for new and efficient methods for numerical computation of electromagnetic scattering and (conventional or fractal) antennas radiation. He is the developer of the graphical electromagnetic computation (GRECO) approach for high-frequency RCS computation, the integral equation formulation of the measured equation of invariance (IE-MEI) and the multilevel matrix decomposition algorithm in 3-D (MLMDA-3D). He has held positions of Visiting Professor at the Swiss Federal Institute of Technology (EPFL), Lausanne, Switzerland, CLUSTER Chair at EPFL, and Visiting Fellow at City University of Hong Kong. He has more than 30 publications in refereed international journals and more than 70 in international conferences.

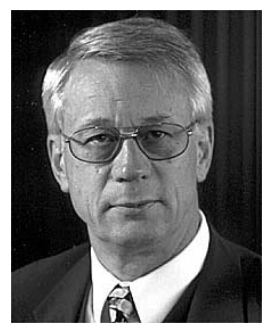

Leo P. Ligthart (M'94-SM'95-F'02) was born in Rotterdam, The Netherlands, on September 15, 1946. He received the Engineer's degree (cum laude) and the Doctor of Technology degree from Delft University of Technology, Delft, The Netherlands, in 1969 and 1985, respectively, and the Doctorates (honoris causa) from Moscow State Technical University of Civil Aviation, Moscow, U.S.S.R., in 1999 and Tomsk State University of Control Systems and Radioelectronics in 2001. He is an academician of the Russian Academy of Transport

Since 1992, he has held the Chair of Microwave Transmission, Radar and Remote Sensing, Department of Information Technology and Systems, Delft University of Technology, where, in 1994, he became Director of the International Research Center for Telecommunications-Transmission and Radar. He has published over 350 papers and one book. His principal areas of specialization include antennas and propagation, radar and remote sensing, but he has also been active in satellite, mobile and radio communications.

Dr. Ligthart is a Fellow of the Institution of Electrical Engineers (IEE), London, U.K.

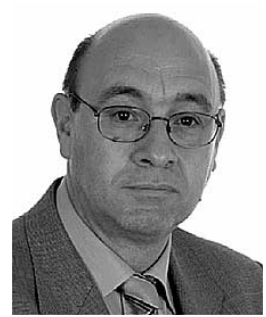

Angel Cardama (S'67-M'73) received the Degree of Telecommunications Engineer from the School of Telecommunications Engineering, Madrid, Spain, in 1968 and the Sc.M. and Ph.D. degrees in electrical engineering from Brown University, Providence, RI, in 1970 and 1973 , respectively.

In 1972, he joined the faculty of the Telecommunication Engineering School, Polytechnic University of Catalonia, Catalonia, Spain, where he holds the position of Professor. From 1987 to 1990, he coordinated the Area of Information and Communications Technologies at the National Agency of Evaluation and Prospective (ANEP), Madrid, Spain. From 1992 to 1996, he was a Member of the Engineering Panel of the Human Capital and Mobility (HCM) and Training and Mobility of Researchers (TMR) Programs of the European Union. Since 1993, he has served as Advisor for Engineering at the Board of Scientific and Technical Assessment of the first three Research Plans for Catalonia. He has been involved in science and technology management and planning at the National and European level. His research interests range from the development of analytical and numerical techniques in electromagnetics to the design of microwave imaging systems and radar and communications antennas.

Dr. Cardama was elected a Member of the Royal Academy of Doctors in 2002. 